\title{
Aqueous two-phase flotation for primary recovery of bacteriocin-like inhibitory substance (BLIS) from Pediococcus acidilactici Kp10
}

\begin{abstract}
An aqueous two-phase flotation (ATPF) system based on polyethylene glycol (PEG) and sodium citrate $(\mathrm{NaNO} 3 \mathrm{C} 6 \mathrm{H} 5 \mathrm{O} 7 \cdot 2 \mathrm{H} 2 \mathrm{O})$ was considered for primary recovery of bacteriocinlike inhibitory substance (BLIS) from Pediococcus acidilactici Kp10. The effects of ATPF parameters namely phase composition, tie-line length (TLL), volume ratio between the two phases (VR), amount of crude load (CL), $\mathrm{pH}$, nitrogen gas flow rate (FR) and flotation time (FT) on the performance of recovery were evaluated. BLIS was mainly concentrated into the upper PEG-rich phase in all systems tested so far. The optimum conditions for BLIS purification, which composed of PEG 8000/sodium citrate, were: TLL of 42.6, VR of 0.4, CL of $22 \%(\mathrm{w} / \mathrm{w}), \mathrm{pH} 7$, average FT of $30 \mathrm{~min}$ and FR of $20 \mathrm{~mL} / \mathrm{min}$. BLIS was partially purified up to 5.9-fold with a separation efficiency of $99 \%$ under this optimal conditions. A maximum yield of BLIS activity of about $70.3 \%$ was recovered in the PEG phase. The BLIS from the top phase was successfully recovered with a single band in SDS-gel with molecular weight of about $10-15 \mathrm{kDa}$. ATPF was found to be an effective technique for the recovery of BLIS from the fermentation broth of P. acidilactici Kp10.
\end{abstract}

Keyword: Aqueous two-phase flotation; Bacteriocin-like inhibitory substance; Pediococcus acidilactici Kp10; Polyethylene glycol; Purification 